Pour faciliter le passage dans los courbes de petit rayon, les essicux de ces deux locomolives ont un jeu latéral.

La ligure 6 représente un lype de locomolive à grande vitesse el de puissance moyenne. Elle possède un bogie porleur à chaque extrémité, el deux essieux porteurs accouplés. Ces derniers sont actionnés par la manivelle d'un arbre intermédiaire commandé à la fois par los bielles obliques de deux moteurs.

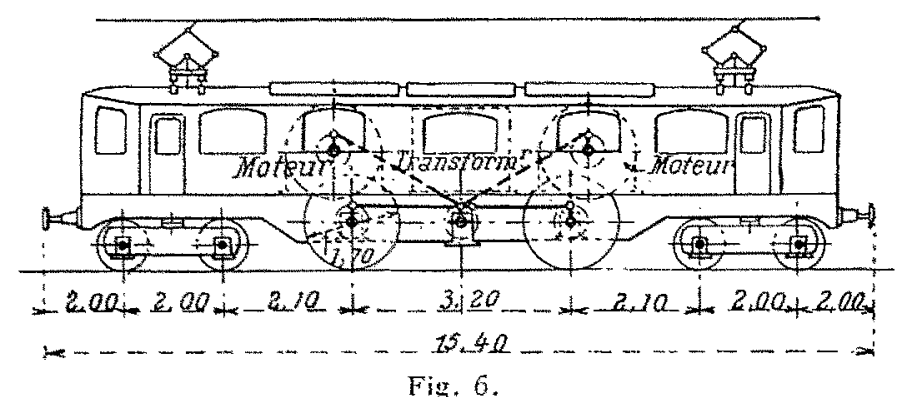

Fig. 6.

Dans toutes ces locomotives, les moteurs el transformaleurs sont disposés aussi près que possible du centre de la machine, of loin des essicux directeurs, de manière à rendre moins sensible l'incrtie des grosses masses fixes el l'effel gyroscopique du mouvement de rolation des induits des moteurs.

Avant de terminer, signalons le type de locomolive électrique monophasé qui vient d'être commandé aux Ateliers d'Oerlikon par la Compagnie du Chemin de fer des Alpes bernoises, concuremment à celles de l'A.F.G., el pour faire le mème service.

Ces machines sont monlées sur deux bogies à trois essieux accouplés, actionnés chacun par un moteur de r ooo HP, avec commande par engrenages et par bielles. Les roues ont $x^{\mathrm{m}} 35$ de diamètre el la longueur totale de la machine est de $14^{\mathrm{m}} ; \mathrm{O}$.

Chaque moleur monophasé est alimenté a 420 volts par un transformateur. L'effort de traction, mesuré à la jante des roues, est de $12800 \mathrm{kgs}$, à la vitesse de $42 \mathrm{~km}$ à l'heure.

L'équipement électrique pèse 42 tonnes, el la machine entière 86 tonnes. La charge de chaque essieu est de 15 tonnes, sauf pour les essieux d'extrémité où elle est limitée à 13 tonnes.

J. C.

\section{INSTALLATTONS HYDR0-ÉLECTRIQUES}

\section{USINE HYORO-ÉLEOTRIQUE DE LA CERVARA}

Le remarquable essor industriel de l'Italie moderne est dû, pour une bonne part, à ce que la presqu'île italienne, fort pauvre en charbon, est, au contraire, très riche en houille blanche, et qu'elle a su en tirer bon parti. D'innombrables usines hydro-électriques ont surgi un peu partout, tant dans les Alpes (\%), que sur cetle longue épine dorsale qu'est l'Apennin. C'est précisément l'une de ces dernières que nous nous proposons de décrire aujourd'hui.

Cette grande station centrale hydro-électrique, qui fournit de l'énergie électrique à l'usine électrochimique de

(*) Voir dans La Houille Blanche de mars el avril j906, la communication de M. Semenza sur les installations hydro-électriques de la Haute Italie.
Narni, a élé inslallée près de Terni (Ombric), dans la valléc de la Néra, afluent du Tibre, par la Società Industriale Lleltrica della Valnerina $\left(^{*}\right)$.

Déjù, en 1886 , cetle société avail fail une première installation pour dislribuer dans Temi la lumière el la force motrice. La première usine utilisail la force molrice du canal de Nerino, et comportait trois alternateurs triphasés de $80 \mathrm{kw}$. Mais l'installation devint bientôt insuffisante $\mathrm{c}$ lon construisit, en 1895 , un second batiment dans lequel furent placées deux génératrices de $85 \mathrm{kw}$ sortant des aleliers de construction d'Oerlikon.

Etant domó le développement industriel très rapide de Temi, on fut amené à rechercher d'autres forces hydrauliques susceptibles de fournir l'énergie nécessaire aux diverses usines de Terni et, en particulier, de son aciérie.

La grande chute de la Cascata delle Marmore, qui s'étend sur une longueur de $7 \mathrm{~km}$. au-dessous de Terni, ct se jette dans la Nera, avait déjà été ubilisée en partie, mais le reste de la concession avait été donné à une autre Compagnie. La Società Industriale Elettrica della Valnerina obtinl alors la concession des chules de la Nera en aval de la Cascata. La hauteur de chule disponible atteint $23 \mathrm{~m}$., le débil moyen de l'eau élant de $40 \mathrm{~m}^{3}$ par seconde, ce qui correspond à une puissance moyenne de 7 ooo kilowalts.

Le projet primitif consistait à hâtir deux slations centrales : une pour l'éclairage et la force molrice de Terni, l'autre pour l'aciéric. Les travaux furent commencés au printemps de rgor. I'inslallation hydraulique fut exéculé tout de suite pour la pleine puissance de la chute, ef l'on construisit une promière station comportant deux turbines à axe horizontal de $\mathrm{x}$ ooo HP, tournant à 250 tours par minute, fournies par la maison Riva et Monneret de Milan. Ces turbines étaient accouplées à deux alternateurs de $865 \mathrm{kw}, 3750$ volts, 42 périodes. On choisit la tension de $37^{50}$ volts, parce que, en montant en étoile les transformateurs à 2 roo volts déjà existants (en triangle), on pouvait utiliser ces transformateurs qui servaient à alimenter le réseau de la ville. Cette installation est en service depuis la fin de rgo3.

La sociélé résolut entre temps d'uliliser toute la puissance disponible pour les besoins de l'usine électro-chimique. Le projet primitif qui comportait deux stations centrales fut donc abandonné, el l'on ne construisit qu'un seul bấliment en rapport avec les nouveaux besoins, dans lequel on installa les deux unités de $r$ ooo HP, primitivement en service.

Aménagement Impraulque. - Limslallation hydraulique ful faite de rgor à xgo3 en aval du pont de Papino. Elle comprend six prises d'eau, de $2 \mathrm{~m}$. de diamètre, munies de vannes en fer, el un eanal à ciel ouvert de seclion trapézö̈dale ayant $385 \mathrm{~m}$. de long, une largeur moyenne de $\mathbf{i} \mathbf{m}$., une profondeur de $2 \mathrm{~m}$. el une pente de $1 \mathrm{~mm}$. par mètre. Go canal débouche dans un bassin de décantation, d'une superficie de $2300 \mathrm{~m}^{2}$, par cinq ouvertures munies d'écluses qui permettent d'isoler entièrement ce bassin dans le cas où des réparations seraient nćcessaires. On a prévu une canalisation de décharge su la Nera comprenant dix conduites de $2 \mathrm{~m}$. de diamètre munies de vannes de fermeture.

Perpendiculairement au bassin, se trouve une murelle dont la crêle arrive à ${ }^{\mathrm{m}} 80$ au-dessous du niveau de l'eau, et qui a pour but d'empêcher les sables et les graviers de

(*) Voir également : Bulletin des Ingénieurs de lTistitut Electrotechnique Montefore 1909 , et aussi La Revue Industrielle IgIg. 
pénélrer dins fes conduiles. Ces subles peuvent elre évacués daus la Nera par des canalisalions munies de vannes.

Deux conduites parallèles, de $520 \mathrm{~m}$. de long, $3^{\mathrm{m}} 5 \mathrm{o}$ de diamètre, partent de ce bassin pour aboulir à la Cervara dans un bassin de mise en charge, de $3640 \mathrm{~m}^{2}$ de superficie. Co bassin esl muni de ra vannes qui permieltent d'isoler les conduiles d'alimentation. Ges vannes avaient été construites conforménent au projel primilif d'apres tequel on devait inslaller r2 unités de r ooo IIP. La nouvelle installation ne prévoyant que 6 unités de $2200 \mathrm{IIP}$, afn d'utiliser les vimnes el les conduites existanles, on réunit par groupes de frois les canalisations partant du bassin de mise on charge (f) facon à leur faire alimenter deux conduites de mise en charge. De cetle façon, neuf de ces vammes desservent les 6 unilés de $2200 \mathrm{HP}$, deux autres vannes servent à l'alimonlalion des deux turbines de s ooo HP déjà existantes, of la douzième alimente les turbines des excilatrices.

les conduiles forcées sont construiles en tòle rivée extradince Sirntens Marlin, d'une épajsseur de $7 \mathrm{~mm}$. I.e dia-

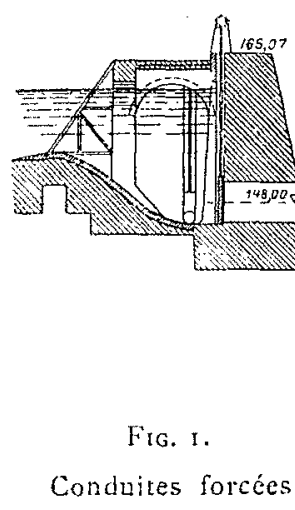

mètre des conduites est de $2^{\text {m }} 30$ pour les grosses turbines, ol de $I^{\mathrm{m}} 7^{5}$ pour les petites. Chaque tuyau est pourvu d'un manchon de dilatation. Chaque canalisation peut être séparée du bassin de mise en charge au moyen d'une vanne d'arrêt spéciale.

Dans une annexe parallèle à la salle des machines, se trouvent des vannes d'arrêt, commandées à l'aide d'appareils hydrauliques que l'on actionne par des volants disposés dans la salle des machines à côté de chaque turbine.

Les turbines, à réaction et à double couronne d'aubes, ont ćté fournies par la maison A. Calzoni de Bologne. Suivant la hauteur de la chule, qui varic de $19^{\mathrm{m}} 60$ a $23^{\mathrm{m}} 7 \mathrm{o}$, chaque lurbine absorbe de 10,3 à $8,7 \mathrm{~m}^{3}$ par seconde, ce qui correspond à une puissance moyenne d'environ a 200 chevanx.

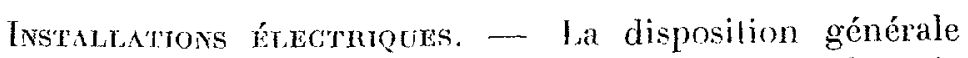
était imposée par les nécessités du service que devait fournir Ia station. Il fallait, d'une part, assures l'éclairage et la distribulion de force motrice de Terni, avec unc tension de 3. To volts, el, d'autre part, alimenter la canalisation électrique à 27 ooo volls qui foumit a Narni l'énergic nécessaire pour l'usine de carbure de calcium. On a prévu à cet difel deux systènes de barres onmibus cntièrement indépendints l'un de l'autre.

Ia salle des machines comprend cinc allernaleurs de r goo kw. directement accouplés aux lurbines de s soo HP, on a en oulre réservé l'emplacement d'une sixième unité. Chacun des alternaleurs peul être réuni à l'ajde d'un conjoncleur-disjoncteur arec chacun des systèmes de barres omnibus. Ics deux pelits allemateurs de $865 \mathrm{kw}$. prove- nant de l'ancienme stalion centrale servent exclusivement à alimenter le róseau de Terni.

Quand les alternateurs travaillent sur le système de barres omnibus à haute tension à 27 ooo volts, pour le service de Narni, ils sont réunis dixeclemenl à un transformatcur de même puissance avec lequel ils forment une unité. Les bornes à haute tension des transformateurs sont reliées aux barres omnibus à 27 ooo volts. On a prévu deux systèmes de barres omnibus, dont l'un est utilisé en service normal, l'aulre servant de réserve. Des conjoncleur's-disjoncteurs permettent d'effectuer les connexions nécessaires entre les transformateurs et les lignes.

L'usine de Narni est reliéc à la station par une double ligne à trois conducteurs. La ligne de Narni est à qualre conducteurs, dont un neutre.

L'excitation des alternateurs s'effectue au moyen de baries omnibus communes à 125 volts ; on a prévu trois machines d'excitation, dont deux sont actionnées par des lurbines particulières d'une puissance de $75 \mathrm{kw}$ tournant à 500 tours. La troisième excitatrice, de $150 \mathrm{kw}$., est actionnéc par un moteur asynchrone de $240 \mathrm{HP}$, tournant à 5oo tours el branché sur les barres omnibus à 3 750 volts. La dynamoshunt directement accouplée au moteur asynchrone domne un courant de 1250 ampères it une tension de 190 ì I5o volts.
La salle des machines a.une longueur de $66 \mathrm{~m}$. et unc largeur de ${ }_{1} 5 \mathrm{~m}$. Sur un des petils còlés sont adossés to bâliment des tableaux de distribution ef le bâtiment des transformateurs. La plate-forme principale de distribulion

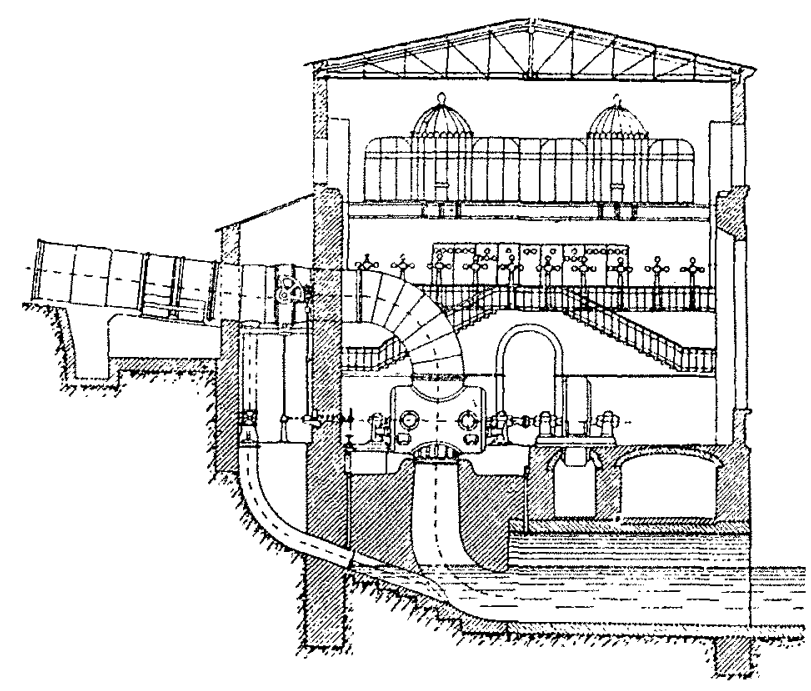

Fla. 2. - Coupe en travers de l'usine génératrice.

est siluée au premier étage do la sallo des machines. Le rez de-chaussée est occupé par les dispositifs de connexion des alternateurs et le rhéostat régulateur principal : an premies étage (qui constitue le rez-de-chaussée du côté extérienr dn bâtiment), se trouvent les transformateurs. Le deuxiòme étage contient les interupteurs el les barres omnilus des 
transformatcurs, le troisième ílage comprend les interupteurs de ligne et les appareils limiteurs de tension.

Les alternateurs donnent uns tension normale de 3750 volts qui peut être pousséc jusquà 4 x5o. Les trois phases sont réunies en étoile. Un qualrième conducteur, fixé à une borne spéciale, serl à assurer l'éclairage de Terni à l'aide de transformateurs monophasés à 2 roo volts.

Les alternateurs sont du type à induil fixe et à inducteur tournant. L'inducteur comporle 20 pôles, et les enroulements sont constitués par des lames de cuivre. Pour obtenir une courbe de tension sinusoïdale aussi régulière que possible, les épanouissements polaires sont construits en forme de gradins. Lcs deux alternateurs de $865 \mathrm{kw}$. sont prévus pour la même tension et le même nombre de lours que les précédents.

Toutes les connexions des excitalrices sont installées dans in couloir facilement accessible situé au-dessus des machines. Les conducteurs à haute lcnsion qui vont des ailernateurs aux interrupteurs sont placés dans un second couloir parallole au premier.

Chaque allernateur comporte un système isolé d'appareils de connexion séparé des autres par une paroi ignifuge et qui comprend : Deux transformateurs d'intensité pour le chacun d'eux, d'unc puissance de 1 goo kw., élève la lension de 3750 à 27000 volts.

Les trois enroulements des transformateurs sont réunis en étoile, et sont enlièrement séparés les uns des aulres. Les conducleurs à basse tension sonl placés près des noyaux, les conductcurs ì haute lension sont disposés à l'extérieur. Les enroulements de haule et de bassc tension sont séparés par des couches de papier imprégné avec interposition de mica. Ces cylindres isolants dépassent en haut et en bas les enroulements d'une quantité lelle qu'aucune décharge n'est à craindre.

Les Iransformateurs sont montés sur roues pour la facilité du transport. Ils comportent des dispositifs de refroidissement par circulation d'air, el sont munis à cet effet d'une enveloppe de tole. Une conduite d'air sous pression dessert tous les transformateurs, ef s'étend immédiatement audessous des appareils. Aux deux extrémités de la conduite se trouve un ventilateur Sulzer actionné par un moteur lriphasé de ro HP ; les ventilateurs sonl calculés de façon à pouvoir assurer chacun un refroidissement suffisant pour lout l'ensemble des transformateurs.

Au second étage, se trouvent les appareils de connexion pour les transformateurs ; ces interrupleurs sont munis do

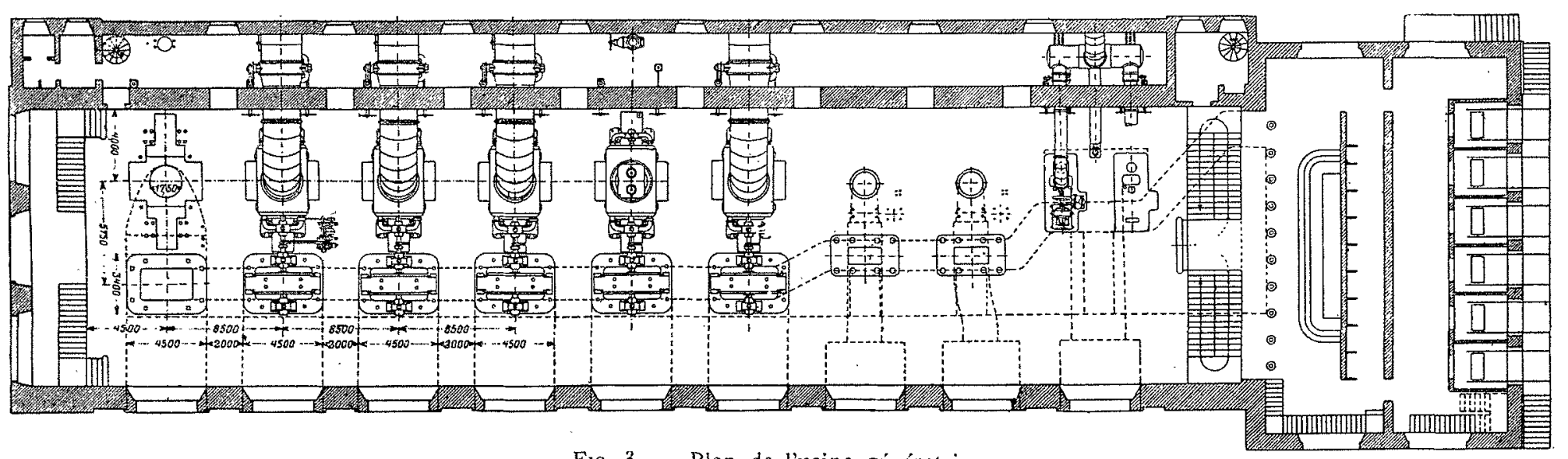

Fig. 3. - Plan de l'usine génératrice.

wallmètre, un pour l'ampèremètre, el trois pour le relais à maximum et à courant de retour, ainsi qu'un transformaleur de tension pour le wattmètre, le relais el le voltmètre.

Sur l'arrière des cellules, dans lesquelles sont renfermés ces groupes d'appareils, est placé un interrupteur à huile télrapolaire, muni de disjoncteurs automatiques à maximum et à courant de retour et de sectionneurs, pour permettre la réunion de l'alternateur avec la barre omnibus à 3750 volts ou le transformateur correspondant. Cet interrupteur à huile est commandé, des colonnes de distribution de la plate-forme principale, à l'aị̂e d'un système de câbles.

Les deux alternateurs de $865 \mathrm{kw}$. sont équipés comme les autres, mais sonl connectés direclement aux barres omnibus à 3750 volts.

Sous les colonnes de distribulion se trouvent les rhéostats régulateurs principanx des alternateurs, avec leurs interrupteurs ì charbon, ainsi que les rhéosiats des excitatrices.

Transformateurs. - Les barres omnibus à 3750 volts conduisent directement le courant du rez-de-chaussée au deuxième élage, où sonl placés les transformateurs dans des cellules isolées adosaćes à l'arrière du bâtiment des tableaux de distribution, et accessibles de l'extérieur. L'installation définilive comportera six cellules, mais actuellement cinc transformateurs seulement sont en service; disjoncteurs automatiques à maxima, ot sonl également commandés des colonnes de distributioin au moyen d'um système de câbles. Tous les organes pour la commande des interrupteurs et la disjonction automatique sont situés audessus des interrupteurs et sont entièrement séparés des conducteurs. Les interrupteurs sont reliés, par l'inlermédiaire de trois transformateurs d'intensité servant à la commande du relais à temps à maxima, à un système de cellules qui comprend les barres omnibus el les disjoncteurs nécessaires.

Barres omnibus, - On a prévu deux systèmes de barres omnibus à 27 ooo volts, l'un de ces systèrnes servant exclusivement de réserve. Des conjoncleur's-disjoncteurs permetlent de réunir les transformateurs el les lignes à l'un des deux systèmes. I.es parois des cellules porlent des écriteaux qui indiquent au personnel quelles sont les connexions quo permellent les différents commutateurs et quelles sont los unités auxquelles appartiennent les différents appareils. Ce système de désignation est appliqué dans lout l'ensemble de l'usine.

Entre les bornes des transformateurs et les feeders, sont intercalés les transformateurs d'intensité et de tension pour les wattmètres, les ampèremètres et vollmètres généraux.

Des conjoncteurs-disjoncteurs permettent de mettre en 
court circuit les barres ommibus, dans le cas où un accident surviendrail aux appareils.

Départ des lignes. - La ligne à 3750 volls pour l'alimentalion de Terni est à 4 conducleurs, dont un conducleur neulre. On a prévu, comme appareils accessoires, trois transformateurs de tension pour les ampèremètres el les relais à maxima et à temps, un interrupleur automatique lélrapolaire à huile, actionné, de la plate-forme principale, par une commande par câbles et une bobine de self pour chaque conducteur.

Les apparcils limiteurs de tension comprennent des parafoudres à cornes avec résistance hydraulique, et des appareils à jet d'eau. On peut vérifier l'isolement de la ligne à l'aide d'un commutateur qui permet de réunir chacun des poles avec une bobine de réaclance, que l'on met à la terre aver inlerposition d'un ohmmètre el d'une pelile batterie Clectrolylique. On peut ainsi s'assurer de l'isolement en surchargeant la ligne. Pour le service intérieur de l'usine, un transformaleur de $60 \mathrm{kw}$., relié aux barres omnibus à 37,50 volts, abaisse la tension de 3750 à 500 volts.

la ligne à 27 ooo volts qui dessert Narni comprend 6 conducteurs. On a prévu pour cetle ligne un interrupleur à huile aulomalique à maxima, qui esl disposé pour être aclionné du tableau de distubulion par l'intermédiaire d'un b́lectro-aimant. Les relais sont actionnés par l'intermédiaire dc trois transformateurs d'intensité, qui servent également i réduire le courant qui traverse les ampèremètres dé ligne. Chacun des conducteurs est équịé avec une bobine d'induclion, el mis en sćrie avec 4 résistances hydrauliques, par l'inlermédiaice d'un parafoudre à cornes et d'un appareil ì jet d'eau, afin d'empêcher loule mise à la terre provenant d'influences athmosphériques ou de surtensions accidenLelles.

Plate-forme de distribulion. - La plate-forme de distribution comprend tous les appareils et tous les instruments de mesure nécessaires au contrôle de l'installation. Les instruments et les leviers de manueurre destinés à la commande des machines sont disposés sur des colonnes ; les tableaux Me distribution sont réservés au contrôle des lignes.

Lés alternateurs de $\mathrm{I}$ goo $\mathrm{kw}$. sont commandés au moyen de 5 colonnes de dislribution qui portent chacune : un levier à main pour commander l'interrupteur automatique ì huile de l'alternateur, un aulre pour l'interrupteur à huile lu transformateur, deux volants pour le rhéostat principaỉ cl l'inlerrupleur à charbon du circuit inducteur. Les interrupleurs de champ et l'interrupleur principal de chaque allernalour sont enclenchés de telle sorte, qu'on ne puisse fermer le circuit de l'alternateur quaprès aroir fermé le circuit d'excilation, et qu'on ne puisse ouvrir le circuit d'excitation qu'après avoir ouvert le circuit de l'alternateur.

Lorsque le circuit de l'allernateur ou du Lransformateur se trouve interrompu par le disjoncteur automatique, les liges de manouvre restent en place, de sorte que l'employé qui surveille le tableau de distribution ne peut pas se rendre compte si l'appareil disjoncteur automatique est fermé ou non ; on a donc prévu un syslème averlisseur à lampesignal.

Les instruments de mesure disposés sur chaque colonne de distribution servant à la commande de chaque alternateu' soul les suivants : Un ampèremètre d'excitation, un impèrcmètre pour l'alternateur, un wattmètre Ferraris, et un double voltmètre pour le couplage en parallèle, permettant d'obtenir une tension uniforme pour les deux ma- chines que l'on veut accoupler. La colonne de distribution porte à cet effet un commutateur à 7 contacts. Du côté de la salle des machines, les colonnes portent une lampe de phase qui sert de signal pour la surveillance des turbines.

Les colonnes de distribution qui commandent les deux alternateurs de $865 \mathrm{kw}$, exclusivement destinés au service de Terni, sont équipées exaclement comme les autres, exception faite du levier de comriande des transformateurs.

Sur une autre colonne de distribution sont groupés les appareils de commande du groupe moleur générateur ; un ampèremètre et un levier de connexion pour le moteur asynchrone, un ampèremètre, un voltmètre, un inicrupleur et un volant pour la manœurre de la dynorno à courant continu. Le rhéostat de mise en marche est placé sur le moleur el commandé directement.

Le contròle des deux excitatrices directement accouplées ivec leur turbine se fait à l'aide d'une même colonne de distribution, au moyen de deux volants, deux leviers, deux atmpèremètres et un voltmètre avec commulateur de voltmètre tripolaire.

A droite et à gauche de la plate-forme de distribution, se trouvent les deux voltmètres génćraux des lignes de Terni et de Narni. Cos roltmètres sont munis de doubles graduations transparentes qui les rendent visibles de la salle des machines.

Le tableau de distribution comporte à sa partie supéricure, de gauche à droite, neufs panneaux :

Le premier panneau est vide pour le moment, el portera après la construction de la seconde ligne de Nami un vollmèlre-enregistreur.

Le second panneau est destiné à la ligne de Narni, el comprend trois ampèremètres, un commulateur pour la commande à distance des interrupteurs à haule tension. Une lampe-signal rouge ct une lampe signal verte complètent l'équipement.

Viennent ensuile les panneaux des tolaliseurs, comprenant trois ampèremètres pour les barres omnibus à 27000 volts et un double wattmètre enregistreur Hartmann et Braun ; puis un panneau pour la vérification de l'isolement, comprenant un ohmmètre, un volant commutaleur pour les divers pôles et un commutateur pour la baltcrie. I. es indications de l'ohmmètre ne sont valables que pour une certaine tension de la batterie, de sorte qu'il est nécessaire, avant une vérification d'isolement, de vérifier par une mesure de contrôle la tension de la batterie. Le cinquième panneau comprend un ampèremètre, un levier à main et des lampessignal pour le transformateur destiné à subvenir aux besoins de l'usine.

On rencontre ensuite les panneaux de commande de la ligne de Terni, les totaliseurs ef inlerrupleurs de feeders pour la vérification de la perte à la terre ; ces appareils sont les mêmes que pour la ligne de Narni. Mais ici, l'interrupteur de ligne est muni d'une commande par câbles.

Le neuvième panneau est un panneau de secours.

A la partic inférieure du panneau de distribution se trourent les relais à temps et à maxima des feeders et des lransformateurs, et les relais à temps et à courant de retour des alternaleurs.

Ligne de transport de force de Narni. - La ligne de Narni a une longueur de $1 / \mathrm{km}$. et comprend 6 conducteurs de $7,2 \mathrm{~mm}$. de diamètre, Les isolateurs, du type Paderno, de la maison Richard Ginori, sont placés sur des lraverses en bois. Les supports des conducteur's sont constitués par des pylônes en fer distants d'une centaine de mètres. I a hauteur 


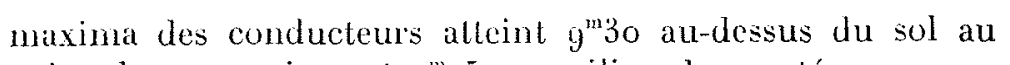
point de suspension, et $\tau^{m} 25$ au milieu des portées.

Station de transformaleurs de Narni. - La station de Iransformaleurs de Narni comprend une tourelle d'amènée du courant, el les batiments de transformateurs.

La lourelle renferme au second étage des parafoudres à cornes et des résistances hydrauliques; au premier étage sont disposées des bobines de self et des appareils à jet d'eau pour les six conducteurs. Un inlerrupteur automatique à huile à maxima est placé au rezde-chaussée, à còlé de trois transformateurs d'intensité el d'un transformateur de tension. Dans le mène local sont réunis, sur un même tableau de marbre, trois ampèremètres, trois voltmètres of un relais bipolaire à maxima.

Le bàliment des transformateurs est constilué par une construction allongée qui comprend d'un còté les cellules des transformaleurs d de l'autre colé, les dispositifs de confròle, dont ils sont séparés par une voie de service. I.es barres omnibus à 27 ooo volts traversent le battiment dans toute sa longueur ; de ces barres partent des branchements qui vont aux cellules des transformateurs. Les cabines de controle comprennent chacune un interrupteur à huile à máxima, ún ampèremètre et un relais à temps avec transformateur d'intensité. Les transformateurs sonl logés dans des cellules séparées ignifuges, avec ventilation artificielle. Les bornes secondaires des transformateurs sont reliées directement aux fours à carbure.

Sur les barres omnibus à 27000 volts est branchée une canalisalion qui sert à alimenter deux transformaleurs pour les besoins intérieurs do la slation, el l'éclairage de la fabrique de carbure.

\section{J. Colliginon,} Ingénieur-Electricien.

\section{AMÉNAGEMENT DES CHUTES}

\section{RÉPARATION DU BARPAGE DE CRAIIPAGNa}

Grampagna est une petile localité du département de l'Ariège qui se trouve sur la rivière du même nom, à quelques kilometres en amont de Pamiers. Flle est surtoul célèbre par les orpailleurs, qui, il n'y a pas bien longlemps, cherchaient cncore le précicux métal dans les alluvions de l'Ariège.

Un pelit barage de dérivation y fut élabli en 1852 au travers de la rivière. A la suile de causes diverses, des érosions se produisirent sur la rive droite de la rivière, le mur d'enracinement du barrage sur celle rive fut contourné par les eaux, ot une partie de l'onvage ful enportée ; dès lors, les caux se précipitèent dans la brèche, rongèrent la rive, et se creusèrent dans le passage ainsi formé un lit assez profond, où passait la tolalité des eaux de la rivière avec une vilesse considérable.

A la suite de cet accident, on tenta la fermeture de la brèche par les méthodes ordinaires, savoir : confection d'un batardeau et réfection des maçomneries à sec à l'abri du batardeau. Mais à la suite d'une crue de l'Ariège, une partie de ce batardeau fut enlevée, et I'on tenta en vain, par la suite, sa reconstruclion.

En présence des incidents qui avaient présidé à la confection du batardeau, et en raison de la violence et de la 